Pacific Journal of Mathematics

SOME AVERAGES OF CHARACTER SUMS 


\section{SOME AVERAGES OF CHARACTER SUMS}

\section{H. WALUM}

Let $\chi$ and $\phi$ be nonprincipal characters $\bmod p$. Let $f$ be a polynomial $\bmod p$ and let $a_{1}, \cdots, a_{p}$ be complex constants. We will assume $a_{j}=a_{k}$ for $j \equiv k(p)$, and thus have $a_{n}$ defined for all $n$. Define

$$
S=\sum_{r} a_{r} \chi(f(r))
$$

and

$$
J_{n}(c)=\sum_{r} \phi(r) \chi\left(r^{n}-c\right),
$$

where the variables of summation run through a complete system of residues $\bmod p$.

The averages in question are

$$
A_{1}=\sum_{a=1}^{p-1}\left|J_{n}(a)\right|^{2}
$$

and

$$
A_{2}=\Sigma|S|^{2},
$$

where the sum in (4) is over the coefficients $\bmod p$ of certain fixed powers of the variables in $f$. Exact formulae for $A_{1}$ will be obtained in all cases, and for $A_{2}$ in an extensive class of cases.

Specifically, the following theorems are true.

THEOREM I. Let $f(r)=y r^{m_{1}}+x r^{m_{2}}+g(r)$ and assume $\left(m_{2}-m_{1}\right.$, $p-1)=1$. Let the sum in (4) be over all $x$ and $y \bmod p$. If $g$ has a nonzero constant term and neither $m_{1}$ nor $m_{2}$ is zero, then

$$
A_{2}=p(p-1) \sum_{r=1}^{p-1}\left|a_{r}\right|^{2}+p^{2}\left|a_{0}\right|^{2} \text {. }
$$

Otherwise,

$$
A_{2}=p(p-1) \sum_{r=1}^{p-1}\left|a_{r}\right|^{2}
$$

THEOREM II. Let $d=(n, p-1), \psi(t)=e^{2 \pi i(r \text { ind }(t) / s)}$, where, naturally, $s \mid(p-1),(r, s)=1$ and $g^{\text {ind }(t)} \equiv t(p)$ for $g$ a primitive root $\bmod p$. If $d s \nmid(p-1)$, then $A_{1}=0$. If $d s \mid(p-1)$ and $\psi \chi^{n}$ is nonprincipal, then $A_{1}=p(p-1) d$. If $d s \mid(p-1)$ and $\psi \chi^{n}$ is principal, then $A_{1}=$ $p(p-1)(d-1)-(p-1)$.

The following is an immediate consequence of the first theorem.

Received November 21, 1963 and in revised form June 16, 1964. Research done under the auspices of the National Science Foundation. 
TheOREM III. Let $f$ be as in Theorem $I$, and assume $\left|a_{r}\right|=1$, $r=1, \cdots, p$. Then there exist $x_{0}, y_{0}, x_{1}$ and $y_{1}$ depending on $\chi$, such that the $S$, as in (1), for $x_{0}$ and $y_{0}$ satisfies $|S|<\sqrt{p}$ and the $S$, for $x_{1}$ and $y_{1}$, satisfies $\sqrt{(p-2)}<|S|$.

Proof of Theorem II. Our principal device is the fact that a function which is periodic $\bmod p$ has a unique expansion by means of the characters $\bmod p[2]$. That is if $h(r)=h(s)$ for $r \equiv s(p)$, then for $n \not \equiv 0(p)$

$$
h(n)=\sum_{\theta} b_{\theta} \theta(n),
$$

where $\theta$ runs through the characters $\bmod p . b_{\theta}$ is given by

$$
(p-1) b_{\theta}=\sum_{r} h(r) \bar{\theta}(r) .
$$

Regarding $J_{n}(c)$ as a periodic function $\bmod p$ of $c$, and expanding $J_{n}(c)$ in the form (7), we obtain, by standard methods,

$$
J_{n}(c)=\sum_{\rho^{n}=\psi^{n}} \pi(\bar{\rho}, \chi) \rho(c)
$$

where $\pi(\alpha, \beta)$ is a Jacobi sum [1]

$$
\pi(\alpha, \beta)=\sum_{r} \alpha(r) \beta(1-r) .
$$

The sum in (9) is over all characters $\rho$ which satisfy the indicated condition.

The expansion (7) has a Parseval identity

$$
\sum_{t=1}^{p-1}|h(t)|^{2}=(p-1) \sum_{\theta}\left|a_{\theta}\right|^{2} .
$$

Thus we can evaluate $A_{1}$ by means of (11) and (9) when we know the value of $|\pi(\alpha, \beta)|^{2}$. Now [1] $|\pi(\alpha, \beta)|^{2}=p$ when $\alpha \neq \varepsilon, \beta \neq \varepsilon$ and $\alpha \beta \neq \varepsilon$, where $\varepsilon$ is the principal character. If $\alpha=\varepsilon$ or $\beta=\varepsilon$, then $|\pi(\alpha, \beta)|^{2}=1$. If $\alpha \beta=\varepsilon$ with $\alpha \neq \varepsilon$ or $\beta \neq \varepsilon$, then $|\pi(\alpha, \beta)|^{2}=p$. By hypothesis, $\chi$ is nonprincipal. Thus $|\pi(\bar{\rho}, \chi)|^{2}$ is $p$ unless $\bar{\rho}=\varepsilon$ or $\bar{\rho} \chi=\varepsilon$. If $\bar{\rho}=\varepsilon$, then $\bar{\rho}=\varepsilon$ and $\psi \chi^{n}$ is principal. If $\bar{\rho} \chi=\varepsilon$, then $\rho=\chi$ and $\rho^{n}=\psi \chi^{n}$ implies $\psi=\varepsilon$ which is excluded by hypothesis. Let $N$ be the number of solutions of $\rho^{n}=\psi \chi^{n}$. If $\psi \chi^{n}$ is nonprincipal then $|\pi(\bar{\rho}, \chi)|^{2}=p$ for all $N$ of the $\rho$ and $A_{1}=p(p-1) N$. If $\psi \chi^{n}$ is principal, then $|\pi(\bar{\rho}, \chi)|^{2}=p$ for $N-1$ of the $\rho$ and $|\pi(\bar{\rho}, \chi)|^{2}=1$ for $\rho=\varepsilon$. Thus, in this case, $A_{1}=(p-1)(p(N-1)+1)=N p(p-1)^{2}$.

$N$, the number of solutions of $\rho^{n}=\psi \chi^{n}$, is the number of solutions of $\sigma^{n}=\psi$. It is a standard lemma from the theory of cyclic groups of order $k$ that $a^{n}=b$ has $(n, k)$ or 0 solutions according to whether 
or not order $b \mid k /(n, k)$. Also, $N$ is the number of solutions of $x^{n}=$ $\psi(g)$, for $x$, in $(p-1)-s t$ roots of unity. From either description of $N$, it follows that $N=d$ or $N=0$ according as $d s \mid(p-1)$ or $d s \nmid(p-1)$, and the theorem follows.

Proof of Theorem I. Referring to the hypotheses of Theorem I,

$$
|S|^{2}=\sum_{r, s} a_{r} \bar{a}_{s} \chi\left(y r^{m_{1}}+x r^{m_{2}}+g(r)\right) \bar{\chi}\left(y s^{m_{1}}+x s^{m_{2}}+g(s)\right)
$$

and thus,

(12) $A_{2}=\Sigma a_{r} \bar{a}_{s} \Sigma \chi\left(y r^{m_{1}}+x r^{m_{2}}+g(r)\right) \chi\left(y s^{m_{1}}+x s^{m_{2}}+g(s)\right)=T_{1}+T_{2}$.

$T_{1}$ is the sum of the terms in (12) such that $r \not \equiv 0$ and $s \not \equiv 0 . T_{2}$ is the sum of the terms in (12) such that $r \equiv 0$ or $s \equiv 0 . T_{1}$ can be witten

$$
T_{1}=\sum_{r \neq 0, s} a_{r} \bar{a}_{s} \chi^{m_{1}}(r / s) A\left(r^{m_{2}-m_{1}}, r^{-m_{1}} g(r) ; s^{m_{2}-m_{1}}, s^{-m_{1}} g(s)\right)
$$

where

$$
A(a, b ; c, d)=\sum_{y+c x+d \neq 0} \chi\left(\frac{y+a x+b}{y+c x+d}\right) .
$$

Now,

$$
A(a, b ; c, d)=\sum_{x} \sum_{y \neq 0} \chi\left(\frac{y+x(a-c)+(b-d)}{y}\right) .
$$

Except when $(a-c) x+(b-d) \equiv 0(p)$,

$$
\sum_{y \neq 0} \chi\left(\frac{y+(a-c) x+(b-d)}{y}\right)=-1 .
$$

Also, $(a-c) x+(b-d) \equiv 0(p)$ when $x \equiv((b-d) /(a-c))(p)$ or when $a \equiv c$ and $b \equiv d$. Thus, if $a \neq \equiv$ or $b \not \equiv d$, then

$$
A(a, b ; c, d)=-(p-1)+p-1=0 .
$$

If $a \equiv c$ and $b \equiv d$, then

$$
A(a, b ; c, d)=p(p-1) .
$$

In view of this (13) becomes the sum over all $r$ and $s$ such that $r \not \equiv 0 \not \equiv s$ and $r^{m_{2}-m_{1}}=s^{m_{2}-m_{1}}, \quad r^{-m_{1}} g(r)=s^{-m_{1}} g(s)$. Since $\left(m_{2}-m_{1}\right.$, $p-1)=1$, we have $r \equiv s$. Thus the sum in (13) is over those $r$ and $s$ such that $r \not \equiv 0 \not \equiv s$ and $r \equiv s$. Thus

$$
T_{1}=p(p-1) \sum_{r=1}^{p-1}\left|a_{r}\right|^{2} .
$$


Now

$$
\begin{aligned}
T_{2}= & \sum_{r \neq 0} a_{r} \bar{a}_{0} \sum_{x, y} \chi\left(y r^{m_{1}}+x r^{m_{2}}+g(r)\right) \bar{\chi}(g(0)) \\
& +\sum_{s \neq 0} a_{0} \bar{a}_{s} \sum_{x, y} \chi(g(0)) \bar{\chi}\left(y s^{m_{1}}+x s^{m_{2}}+g(s)\right) \\
& +\left|a_{0}\right|^{2} \sum_{x, y} \chi(g(0)) \bar{\chi}(g(0))=p^{2}\left|a_{0}\right|^{2}|\chi(g(0))|^{2},
\end{aligned}
$$

except when $m_{1}=0$ or $m_{2}=0$.

Thus, if $g(0) \equiv 0$,

$$
A_{2}=p(p-1) \sum_{r \neq 0}\left|a_{r}\right|^{2}
$$

and if $g(0) \not \equiv 0$, then

$$
A_{2}=p(p-1) \sum_{r \neq 0}\left|a_{r}\right|^{2}+p^{2}\left|a_{0}\right|^{2},
$$

when $m_{1}=0$ or $m_{2}=0$, then $\chi(g(0))$ in (14) must be changed to $\chi(y+g(0))$ or $\chi(x+g(0))$, and $A_{2}$ is given by (6).

\section{REFERENCES}

1. H. Davenport, and H. Hasse, Die Nullstellen der Kongruenzzetafunktionen in gewissen zyklischen Fallen, Journal für Math. (Crelle) 172 (1934), S. 151-182.

2. M. J. Delsarte, Essai sur l'application de la theorie des fonctions periodiques a l'rithmetique, Annales Scientifiques L'Ecole Normale Superieure, series 3, 62 (1945), 185204.

Ohio State University 


\section{PACIFIC JOURNAL OF MATHEMATICS}

EDITORS

H. SAMELSON

Stanford University

Stanford, California

R. M. BLUMENTHAL

University of Washington

Seattle, Washington 98105
*J. DUGUNDJI

University of Southern California

Los Angeles, California 90007

RICHARD ARENS

University of California

Los Angeles, California 90024

\section{ASSOCIATE EDITORS}

E. F. BECKENBACH

B. H. NeumanN

F. WOLF

K. YosidA

\section{SUPPORTING INSTITUTIONS}

UNIVERSITY OF BRITISH COLUMBIA

CALIFORNIA INSTITUTE OF TECHNOLOGY

UNIVERSITY OF CALIFORNIA

MONTANA STATE UNIVERSITY

UNIVERSITY OF NEVADA

NEW MEXICO STATE UNIVERSITY

OREGON STATE UNIVERSITY

UNIVERSITY OF OREGON

OSAKA UNIVERSITY

UNIVERSITY OF SOUTHERN CALIFORNIA
STANFORD UNIVERSITY

UNIVERSITY OF TOKYO

UNIVERSITY OF UTAH

WASHINGTON STATE UNIVERSITY

UNIVERSITY OF WASHINGTON

AMERICAN MATHEMATICAL SOCIETY CHEVRON RESEARCH CORPORATION

TRW SYSTEMS

NAVAL ORDNANCE TEST STATION 


\section{Pacific Journal of Mathematics}

\section{Vol. 16, No. $1 \quad$ November, 1966}

Larry Armijo, Minimization of functions having Lipschitz continuous first

partial derivatives ............................... 1

Edward Martin Bolger and William Leonard Harkness, Some

characterizations of exponential-type distributions.............. 5

James Russell Brown, Approximation theorems for Markov operators ...... 13

Doyle Otis Cutler, Quasi-isomorphism for infinite Abelian p-groups ...... 25

Charles M. Glennie, Some identities valid in special Jordan algebras but not valid in all Jordan algebras .......................... 47

Thomas William Hungerford, A description of $\operatorname{Mult}_{i}\left(A^{1}, \cdots, A^{n}\right)$ by

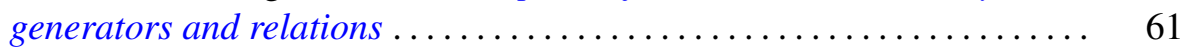

James Henry Jordan, The distribution of cubic and quintic non-residues ....

Junius Colby Kegley, Convexity with respect to Euler-Lagrange differential operators................................... 87

Tilla Weinstein, On the determination of conformal imbedding ......... 113

Paul Jacob Koosis, On the spectral analysis of bounded functions ........ 121

Jean-Pierre Kahane, On the construction of certain bounded continuous functions ................................... 129

V. V. Menon, A theorem on partitions of mass-distribution ........... 133

Ronald C. Mullin, The enumeration of Hamiltonian polygons in triangular

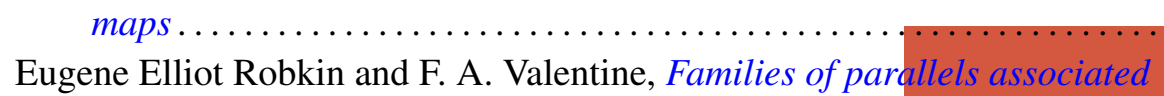

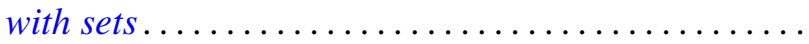

Melvin Rosenfeld, Commutative F-algebras

A. Seidenberg, Derivations and integral closure

S. Verblunsky, On the stability of the set of exponents of a Cauchy

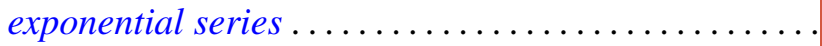

Herbert Walum, Some averages of character sums 